\title{
Impact of the coxsackievirus and adenovirus receptor on the adenoma-carcinoma sequence of colon cancer
}

\author{
K Stecker', M Vieth², A Koschel', B Wiedenmann', C Röcken ${ }^{3}$ and M Anders*,4 \\ 'Department of Internal Medicine, Divisions of Gastroenterology and Hepatology, Charité Medical School, Campus Virchow, Augustenburgerplatz I, \\ Berlin 13353, Germany; ${ }^{2}$ Institute of Pathology, Klinikum Bayreuth, Preuschwitzer Str. 101, Bayreuth 95445, Germany; ${ }^{3}$ Institute of Pathology, \\ Christian-Albrechts-University, Kiel, Arnold-Heller-Str. 3, Kiel 24 105, Germany; ${ }^{4}$ Department of Interdisciplinary Endoscopy, University Hospital \\ Hamburg Eppendorf, Martinistr. 52, Hamburg 20246, Germany
}

BACKGROUND: Coxsackie and adenovirus receptor (CAR) has been suggested to function as a tumour suppressor. Its impact on the adenoma-carcinoma sequence of the colon, however, is unclear.

METHODS: Coxsackie and adenovirus receptor was analysed in non-cancerous and neoplastic colon samples using immunohistochemistry and quantitative RT-PCR. The function of CAR in colon cancer cell lines was determined following application of CAR siRNA or ectopic expression of a human full-length CAR cDNA.

RESULTS: Compared with healthy mucosa, increased CAR-mRNA expression was found in adenomas, whereas primary cancers and metastases displayed a marked decline. At the plasma membrane, CAR was present in normal mucosa samples (93\%), adenomas, and metastases (100\% ea.), whereas in colon cancers, it was found less frequently $(49 \%, P<0.0001)$. Cytoplasmic CAR immunopositivity increased from normal mucosa $(22 \%)$, to adenomas $(73 \%, P=0.0006)$, primary cancers $(83 \%, P<0.000 \mathrm{I})$, and metastases $(67 \%, P=0.0019)$. In cancer cell lines, CAR inhibition resulted in increased proliferation, whereas enforced ectopic CAR expression led to opposite results. Blocking the extracellular portion of CAR increased cell invasion in vitro. In mice, xenotransplants of colon cancer cells with enforced CAR expression formed significantly smaller tumours, whereas CAR inhibition increased the formation of liver metastases.

CONCLUSION: We conclude that CAR facilitates complex effects during colon carcinogenesis, potentially mediated by its stagedependent subcellular distribution; high CAR expression potentially prevents apoptosis in adenomas, loss of CAR at the plasma membrane promotes growth, and dissemination of primary cancers, and high membranous CAR presence may support the establishment of distant metastases.

British Journal of Cancer (201 I) I 04, 1426- 1433. doi:10.1038/bjc.2011.116 www.bjcancer.com

Published online 5 April 20II

(c) 201 I Cancer Research UK

Keywords: coxsackie adenovirus receptor; adenoma-carcinoma sequence; colon cancer

Colon cancer represents one of the leading cancer entities worldwide (Parkin et al, 2005). It most commonly develops from adenomas, referred to as 'adenoma-carcinoma sequence' (Cho and Vogelstein, 1992). The clinical outcome of colon cancer is critically determined by local tumour growth, as well as by the presence of local and distant metastases. For both, invasion and metastatic spread, an impaired adhesion of cancer cells is considered as a crucial prerequisite. Although previously studied, particularly for the adherens junction protein E-cadherin (Buda and Pignatelli, 2004), investigation of tight junctions (TJs) in colon cancer has turn out to be of interest in recent years. Tight junctions represent a complex of proteins in epithelial and endothelial cells separating the apical and basolateral plasma membrane (Satoh et al, 1996; Tsukita et al, 1996). They maintain cellular polarity as well as tissue integrity, and regulate the paracellular transport (Tsukita and Furuse, 2000). An impaired TJ barrier has been found in human colon adenomas and in the

*Correspondence: Dr M Anders; E-mail: m.anders@uke.uni-hamburg.de Revised 2I February 201 I; accepted I2 March 20I I; published online 5 April 2011 'pre-neoplastic' colon of rats treated with the carcinogen 1 , 2-dimethylhydrazine (DMH) (Soler et al, 1999). In colon cancer, a decreased presence of TJ proteins has been described for instance for ZO-1, occludin, and claudin 8, in part, correlating with poor cancer differentiation, disease recurrence, and an adverse prognosis (Kimura et al, 1997; Resnick et al, 2005; Grone et al, 2007). In contrast, TJ protein upregulation in colon cancers compared with normal colon mucosa has been noted for instance for claudins 1 and 12 (Miwa et al, 2000; Grone et al, 2007). These observations point to a complex dysregulation of TJ proteins in colon carcinogenesis. The functional role of this phenomenon, however, has rarely been investigated: Claudin 1 has been shown to induce epithelial-mesenchymal transdifferentiation in cultured human colon cancer cell lines, to promote the growth of xenograft tumours and the formation of metastases in athymic mice (Dhawan et al, 2005). A more detailed understanding of the function of TJs in colon cancer, however, is still missing.

The coxsackie adenovirus receptor (CAR), a transmembrane glycoprotein, was initially characterised as a viral attachment site on the surface of epithelial cells (Bergelson et al, 1997). Later on it was identified as a component of the TJ complex, an interacting 
partner for a number of $\mathrm{TJ}$ proteins, and a regulator of $\mathrm{TJ}$ formation (Cohen et al, 2001; Sollerbrant et al, 2003; Coyne et al, 2004; Excoffon et al, 2004; Mirza et al, 2005; Raschperger et al, 2006). In the context of malignant diseases, reduced CAR presence was found in several solid cancers partially associated with poor differentiation, increased infiltration, and an unfavourable clinical outcome (Rauen et al, 2002; Sachs et al, 2002; Matsumoto et al, 2005; Korn et al, 2006; Buscarini et al, 2007; Okegawa et al, 2007; Anders et al, 2009b). In primary colon cancers, a high variability in CAR expression was found, with approximately $75 \%$ of the cases showing CAR downregulation in (Zhang et al, 2008). Functionally, loss of CAR has been suggested to weaken intercellular adhesion, to increase proliferation, and to promote migration as well as invasion of cancer cells (Okegawa et al, 2000, 2001; Bruning and Runnebaum, 2003, 2004; Huang et al, 2005; Wang et al, 2005; Anders et al, 2009a,b). Recently, we demonstrated that downregulation of $\alpha$-catenin following reduced expression of CAR substantially contributes to these phenomena (Stecker et al, 2009). On the basis of these findings, a tumour-suppressive role of CAR in human cancers has been postulated. In contrast, it has also been speculated that CAR advances the development of adenocarcinomas, as elevated CAR expression was found in early-stage breast and oesophageal cancer (Anders et al, 2003b; Kuster et al, 2010). Moreover, it has been demonstrated that CAR protects adenocarcinoma cells against apoptosis and is needed for efficient tumour formation (Qin et al, 2004; Bruning et al, 2005).

Prompted by these findings, suggestive of a complex role of CAR in carcinogenesis, we aimed to clarify the influence of CAR on the pathobiology of colon cancers. We determined CAR presence in the course of the adenoma-carcinoma sequence and studied CARs function in vitro and in vivo using cultured colon cancer cell lines, following ectopically regulated CAR expression.

\section{MATERIALS AND METHODS}

\section{Study population and tissues}

Tissue samples were obtained from 82 patients (43 men, mean age 70 years; range: 43-91 years) comprehending normal colon mucosa $(n=27)$, tubular adenomas $(n=22)$, primary colon adenocarcinomas $(n=53)$, and metastatic colon cancers (total $n=22$ : liver $n=6$, lung $n=4$, lymph nodes $n=12$ ). Staging and diagnosis was assessed according to the WHO classification (Hamilton, 2000) and the TNM staging, set out by the International Union against cancer (Wittekind, 2003).

\section{Quantitative mRNA determination}

Total RNA was isolated from three consecutive $15-\mu \mathrm{m}$ tissue sections containing at least $80 \%$ of the tissue of interest using the ABSOLUTELY RNA FFPE KIT (Stratagene, Amsterdam, The Netherlands) according to the manufacturer's protocol. Upon reverse transcription of $50 \mathrm{ng}$ of total RNA with random hexamer primers using the 'first-strand synthesis system for RT-PCR' (Invitrogen, Karlsruhe, Germany), real-time quantification was carried out as described previously (Anders et al, 2003b) on a Stratagene MX3000P cycler. Quantification was assessed by the comparative $\Delta C_{\mathrm{T}}$ method normalising $C_{\mathrm{T}}$ values to $\beta$-actin. Complementary DNA derived from Chinese hamster ovary cells (CHO, CHO-CAR; a kind gift of Dr J Bergelson, Division of Infectious Diseases, Children's Hospital of Philadelphia, Philadelphia, PA, USA) was used as negative and positive control, respectively.

\section{Immunohistochemistry}

Immunohistochemical staining was carried out as described previously (Anders et al, 2009b). In brief, tissue sections were deparaffinised, rehydrated, and submitted to antigen retrieval by microwave treatment. Anti-CAR (H-300: sc-15405, Santa Cruz Biotechnology, Inc., Santa Cruz, CA, USA; $1: 50$ ) served as primary antibody. A biotinylated goat anti-rabbit immunoglobulin (Vector Laboratories, Burlingame, CA, USA; $1: 400$ ) was used as secondary antibody, followed by treatment with streptavidin-biotinylated horseradish peroxidase complex (Vectastain Elite $\mathrm{ABC}$ kit, Vector Laboratories). Using diaminobenzidine tetrahydrochloride (Sigma-Aldrich, Munich, Germany), sections were developed and counterstained with haematoxylin. Staining results for CAR were evaluated by estimating the percentage of epithelial cells showing specific immunoreactivity by an expert pathologist (MV) who was blinded for the clinical data. Immunopositivity at the plasma membrane and in the cytoplasm was assessed separately. The CAR status was classified as: negative (no immunoreactivity), weak ( $0-5 \%$ positive cells), moderate $(5-50 \%$ positive cells), or strong ( $>50 \%$ positive cells). Only samples showing moderate or strong immunoreactivity were considered positive.

\section{Cell culture and generation of stably transfected cell lines}

Human colon cancer cell lines SW480, SW620, DLD1, and HCT116 were obtained from the American Type Culture Collection (Rockville, MD, USA) and from the 'Deutsche Sammlung von Mikroorganismen und Zellkulturen GmbH' (Braunschweig, Germany), respectively, and cultured in the recommended growth media. Chinese hamster ovary cells were cultured in Ham's F12 containing 10\% FCS. Colon cancer cell lines with functional CAR knocked down by specific CAR siRNAs or ectopic expression by human full-length CAR cDNA expressed under control of the CMV promoter in a pcDNA3.1 expression vector ('hCARpcDNA3.1'; a kind gift of Dr J Bergelson), were generated as described previously (Anders et al, 2009b). Expression of CAR in pooled cell populations was determined by western blotting, and FACS was used for the assessment of CAR presence at the cell surface.

\section{Western blotting}

Protein lysates were obtained as previously described (Anders et al, 2003a). Subsequently, equal amounts of protein lysates were loaded on reducing Laemmli gels, immunoblotted with specific antibodies against CAR (H-300: sc-15405, Santa Cruz Biotechnology), or $\beta$-actin (Sigma-Aldrich), and detected using the ECL system (Amersham Pharmacia, Piscataway, NJ, USA). Protein lysates of $\mathrm{CHO}$ and $\mathrm{CHO}-\mathrm{CAR}$ cells were used as negative and positive controls, respectively.

\section{Measurement of CAR cell surface expression}

Expression of CAR cell surface was analysed as previously described (Anders et al, 2003a). In brief, living cells were incubated with the anti-CAR antibody RmcB (a kind gift of Dr J Bergelson) in binding buffer (PBS containing 2\% BSA and 10\% normal goat serum) at $4{ }^{\circ} \mathrm{C}$ for $45 \mathrm{~min}$. Afterwards, cells were incubated with a Cy-3-conjugated anti-mouse antibody (Molecular Probes, Eugene, OR, USA). Analysis of stained cells was performed on a FACSCalibur cytometer (Becton Dickinson, Franklin Lakes, NJ, USA).

\section{Assessment of colon cancer cell proliferation in vitro and in vivo}

Cells were seeded onto six-well plates ( $n=3 \times 10^{5}$ cells per well) in the recommended growth media containing $10 \%$ FCS. After $48 \mathrm{~h}$, cells were detached using trypsin and counted using a haematocytometer (VWR International, Darmstadt, Germany). All experiments were performed in triplicate and repeated at least twice. To determine colon cancer cell growth in vivo, $10^{7}$ cells were injected subcutaneously into both flanks of 6- to 8-week-old male NOD/ SCID mice obtained from The Jackson Laboratory (Bar Harbor, 
ME, USA). Tumour volumes were measured twice per week from day 8 onwards with a calliper in two dimensions and calculated according to volume $=$ length $\times$ wide $^{2} / 2$. All animal experiments were performed according to German law at the 'Experimental Pharmacology \& Oncology GmbH (Berlin-Buch, Germany).

\section{Cell invasion into matrigel}

Cells cultured in media with or without the anti-CAR antibody $\mathrm{RmcB}$ (a kind gift of Dr J Bergelson) were seeded onto the top of 'BioCoat Matrigel Invasion Chambers' (BD Biosciences, Bedford,

\section{A}

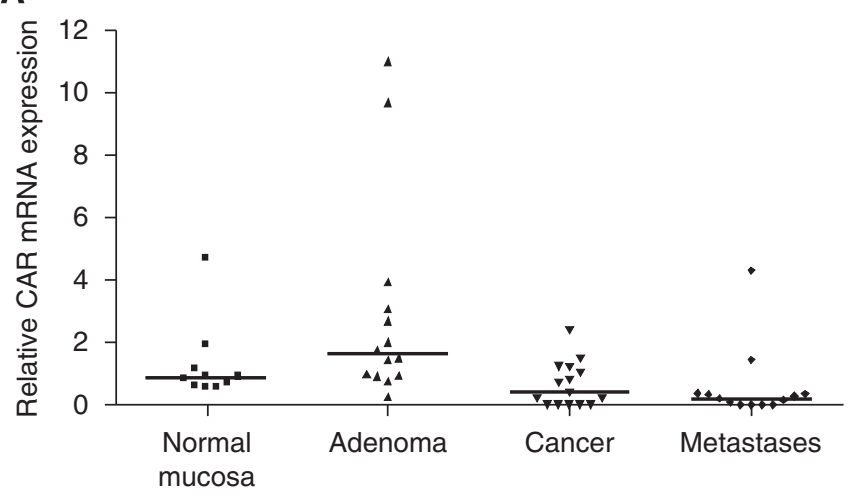

B

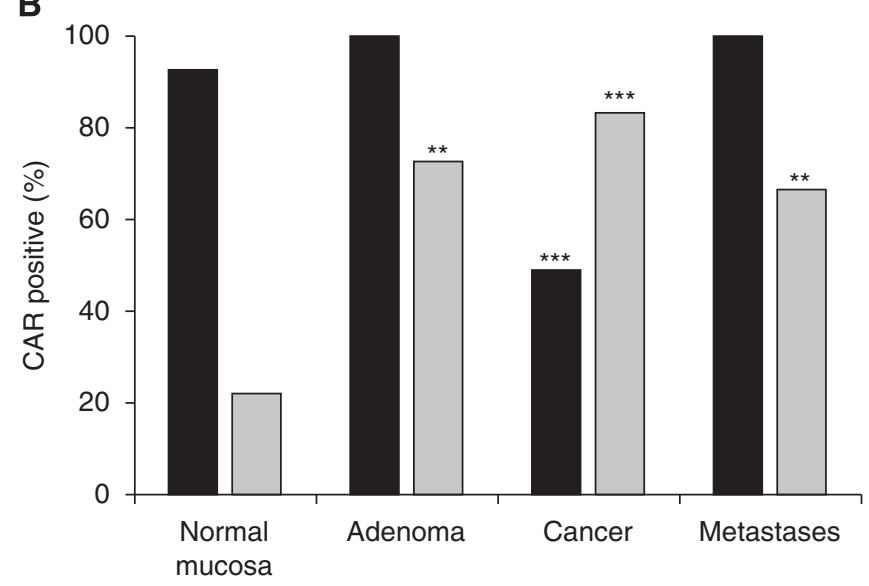

C
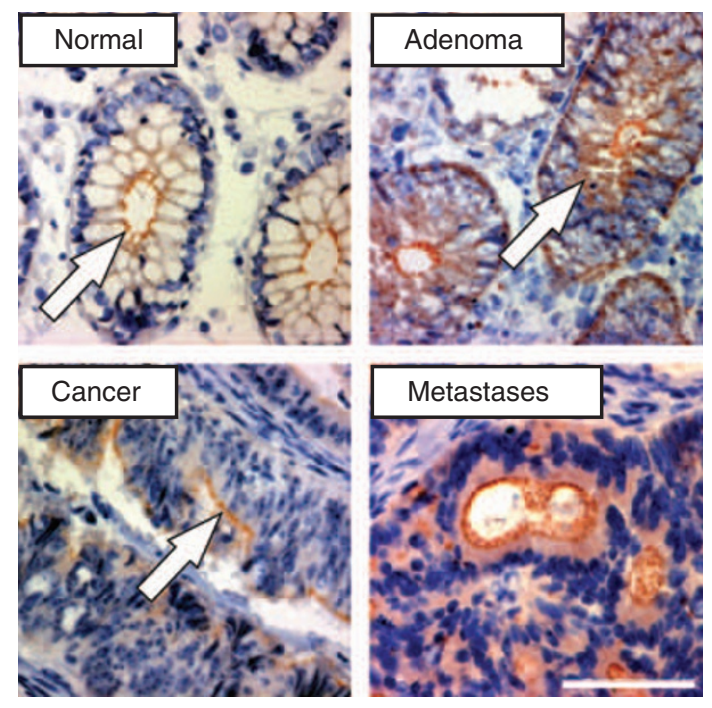

MA, USA) containing $8-\mu \mathrm{m}$ pore size PET membranes covered with matrigel matrix. Medium containing $10 \%$ FCS was added to the bottom well of the chambers as a chemoattractant, whereas serum-free medium was used as a control. Following $48 \mathrm{~h}$ at $37^{\circ} \mathrm{C}$ and $5 \% \mathrm{CO}_{2}$, cells invaded the matrigel-coated membrane, located at the lower membrane surface, were fixed, and stained by crystal violet containing $10 \%$ ethanol. Cells of three representative areas in each well were counted at a magnification of $\times 100$. Experiments were performed in triplicate and repeated at least twice.

\section{Colon cancer cell metastasis in vivo}

The impact of CAR inhibition on the metastatic behaviour of colon cancer cells was assessed in 6-week-old female athymic SCID mice obtained from Charles River Laboratories (Sulzfeld, Germany). All mice received injections of $1 \times 10^{6}$ cells (SW480 following CAR inhibition, SW620 with CAR upregulation or the respective controls) into the spleen. At 4 weeks, all mice were killed and spleen, liver, lung, and paraaortic lymph nodes were obtained, fixed in formalin, and embedded in paraffin. Subsequent histopathological analyses were undertaken using haematoxylin and eosin stained sections. All animal experiments were approved by the local governmental authorities (Landesamt für Gesundheit und Soziales, Berlin, Germany).

\section{Induction of apoptosis}

To assess caspase activity, colon cancer cells were grown in $1 \times 10^{4}$ 96-well culture plates. After $24 \mathrm{~h}$ of incubation, apoptosis was induced by addition of $50 \mathrm{ng} \mathrm{ml}^{-1}$ TRAIL (Biomol, Hamburg, Germany). Following another $48 \mathrm{~h}$, caspase- $3 / 7$ activity was determined using Caspase-Glo 3/7 Assay Systems (Promega, Mannheim, Germany) at 1,2 , and $3 \mathrm{~h}$ after addition of Caspase-Glo 3/7 on a luminescence reader (Mithras LB 940, Berthold Technologies GmbH \& Co. KG, Bad Wildbad, Germany). Hereafter, cell numbers were assessed photometrically following addition of crystal violet (Spectramax 340 PC Microplate Reader, Molecular Devices, Sunnyvale, CA, USA). All experiments were performed in triplicate and repeated at least twice.

\section{Statistical analysis}

Statistical calculations were performed with the GraphPad Prism software (version 4.00; GraphPad Software, Inc., San Diego, CA, USA) using the $t$-test or $\chi^{2}$-test, when applicable.

Figure I Presence and distribution of CAR in non-transformed colon mucosa, adenomas, primary, and metastatic colon cancers. Real time RT-PCR data represent median CAR mRNA expression level of nontransformed mucosa, adenomas, primary colon cancers, and metastases displaying highest CAR expression in adenomas (A). Immunohistochemistry revealed abundant CAR presence at the plasma membrane (black bars) in non-transformed mucosa samples, adenomas, primary colon cancer, and metastases. In colon cancers, a significant decrease of membranous CAR immunoreactivity was found. Cytoplasmic CAR immunopositivity (grey bars) was observed significantly more often in adenomas, primary colon cancers, and metastases (asterisks mark significant differences compared with normal mucosa) (B). Typical results for CAR immunostaining are shown in non-transformed colon mucosa, displaying CAR localisation at the apical plasma membrane (arrow), whereas in adenomas additional cytoplasmic CAR immunoreactivity was noted (arrow). CAR immunoreactivity in primary colon cancers was preferentially seen in the cytoplasm (arrow), whereas CAR presence at plasma membrane was frequently lost. In contrast, metastases displayed both membranous and cytoplasmic CAR presence $(\mathbf{C})$ (magnification: $\times 100$, bar $=200 \mu \mathrm{m})$. 


\section{RESULTS}

Differential abundance and subcellular distribution of CAR in colon tissue

We first studied the CAR mRNA expression by quantitative $\mathrm{RT}-\mathrm{PCR}$ assays on randomly selected tissue specimens of normal colon mucosa $(n=10)$, adenomas $(n=14)$, primary colon cancers $(n=15)$, and colon cancer metastases $(n=13)$. These studies showed that the median $\Delta C_{\mathrm{T}}$ values for CAR mRNA in comparison with normal mucosa $(0.8805)$ increased significantly in adenomas (1.621; $P=0.049$ ), whereas a decrease of CAR mRNA expression was found in primary colon cancers $(0.3920 ;$ n.s. $)$ and colon cancer metastases $(0.2040 ; P=0.032)$ (Figure 1A).

Using immunohistochemistry, we then analysed the presence and histoanatomical distribution of CAR. On a subcellular level, CAR was found at the plasma membrane of normal mucosa samples (93\%), as well as adenomas and colon cancer metastases (100\% each). Interestingly, membranous CAR immunoreactivity was found significantly less commonly in primary colon cancers $(49 \%, P<0.0001)$. In contrast, the prevalence of cytoplasmic CAR immunopositivity was increased in adenomas $(73 \%, P=0.0006)$, primary colon cancers $(83 \%, P<0.0001)$, and metastases $(67 \%$, $P=0.0019)$, compared with non-neoplastic mucosa (22\%) (Figures $1 \mathrm{~B}$ and $\mathrm{C})$. For a subset of 11 patients, we were able to directly compare CAR presence in primary cancers and metastasis. Primary cancers were either entirely CAR negative ( $n=4$ patients) or displayed a moderate CAR immunopositivity (mean $=40 \%$ CAR-positive cells). In contrast, metastatic cancers of all patients were CAR immunopositive.

To assess whether CAR presence and/or subcellular distribution in colon cancer correlates with clinico-pathological parameters, we compared the CAR transcription (mRNA levels) and translation (immunoreactivity) with local tumour growth (T-category), nodal status (N-category), distant metastases (M-category), tumour grade (G), lymph (L-category) and blood-vessel-invasion (V-category), and the UICC tumour stage. None of these parameters correlated significantly with the expression and presence of CAR in primary colon cancers (data not shown).

\section{CAR expression in colon cancer cell lines}

In permanent colon carcinoma cell lines, FACS revealed the presence of CAR in all tested cell lines, with comparable CAR surface manifestation in DLD1, HCT116, and SW480, however, markedly lower levels in the SW620 cell line (Figure 2A). On the basis of these findings, we chose all cell lines for both CAR downregulation and forced expression. Western blotting confirmed reduced CAR protein expression in all cell lines after stable transfection of a CAR-specific siRNA and increased CAR protein levels following ectopic expression of hCARcDNA (Figure 2B). Presence of CAR at the surface in these cell lines was assessed by FACS in unfixed cells. Upon CAR inhibition, a moderate decrease was noted in the DLD1 cell line and, to a less extent, in HCT116 and SW480. In SW620 cells, no differences were noted. Following stable expression of CAR, full-length cDNA, a more than 2.5-fold increase in CAR surface presence was found in DlD1 and SW480 cells, whereas in HCT116 and SW620, a moderate increase was only found (Figure $2 \mathrm{C}$ ).

\section{CAR inhibits growth of colon carcinoma cell lines in vitro} and in vivo

To clarify the influence of CAR on the growth of colon cancer cells, we performed in vitro proliferation assays following ectopic regulation of CAR expression. The RNAi-mediated functional CAR knockdown resulted in significantly higher cell numbers in DLD1 and HCT116 compared with vector controls, whereas for
A

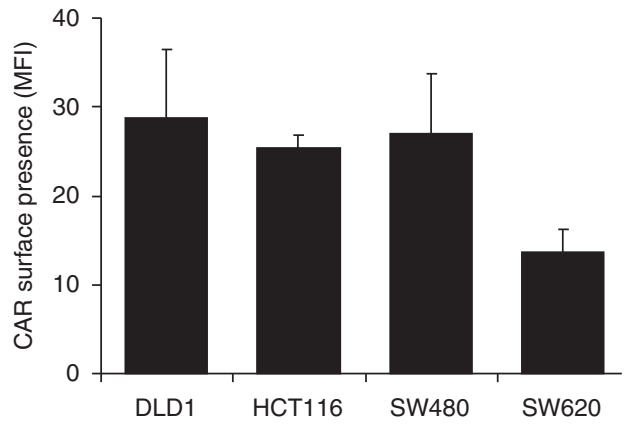

B

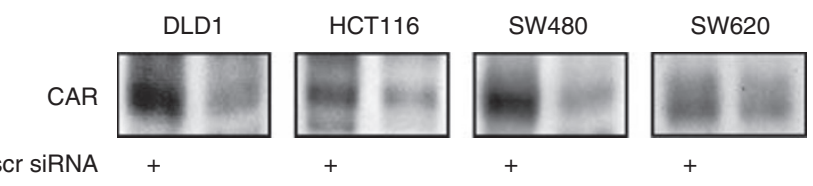

CAR SIRNA
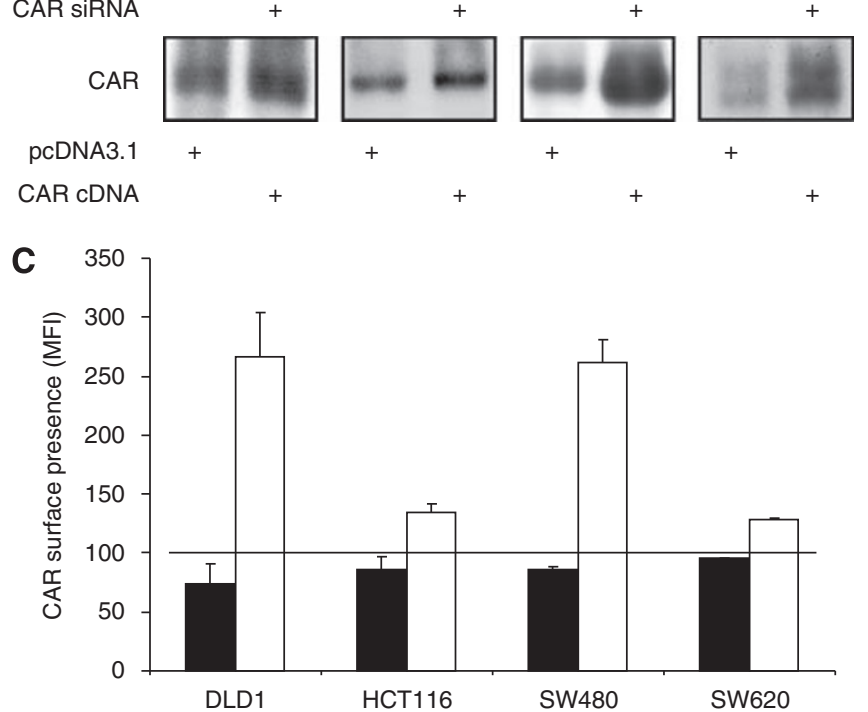

Figure 2 CAR expression and ectopic regulation in colon cancer cell lines. The presence of CAR at the surface was determined by FACS. Data represent the difference between mean fluorescence intensities (MFI) measurements for the anti-CAR antibody $\mathrm{RmcB}$ and a control containing the secondary antibody only $(\mathbf{A})$. To regulate CAR expression in colon cancer cell lines, stable transfections with either a CAR-specific siRNA (upper panels) or a human full-length CAR expression vector 'hCARpcDNA3. I' (lower panels) and the respective controls (scrambled [scr] siRNA or pcDNA3.I) were performed. Differential CAR protein expression was assessed by western blotting (B) and CAR surface presence was determined by FACS $(\mathbf{C})$.

SW480 and SW620, a minor insignificant increase was found. Ectopic CAR upregulation resulted in a significant decline of cell numbers in SW480, SW620, and DLD1 compared with matching controls, whereas for the HCT116 cell line, no significant differences were noted (Figure 3). Subcutaneous xenograft tumours of colon cancer cell lines displayed a markedly reduced tumour size upon ectopic CAR upregulation compared with 'vector only' controls (SW480 $(P=0.02)$ (Figure 4) and DLD1 $(P=0.11)$ (data not shown) $)$.

\section{CAR inhibition increases invasion of colon carcinoma cells}

The impact of CAR on invasion of colon cancer cells following blockade of the extracellular portion of CAR was assessed using an in vitro assay. Incubation with the anti-CAR antibody $\mathrm{RmcB}$, 

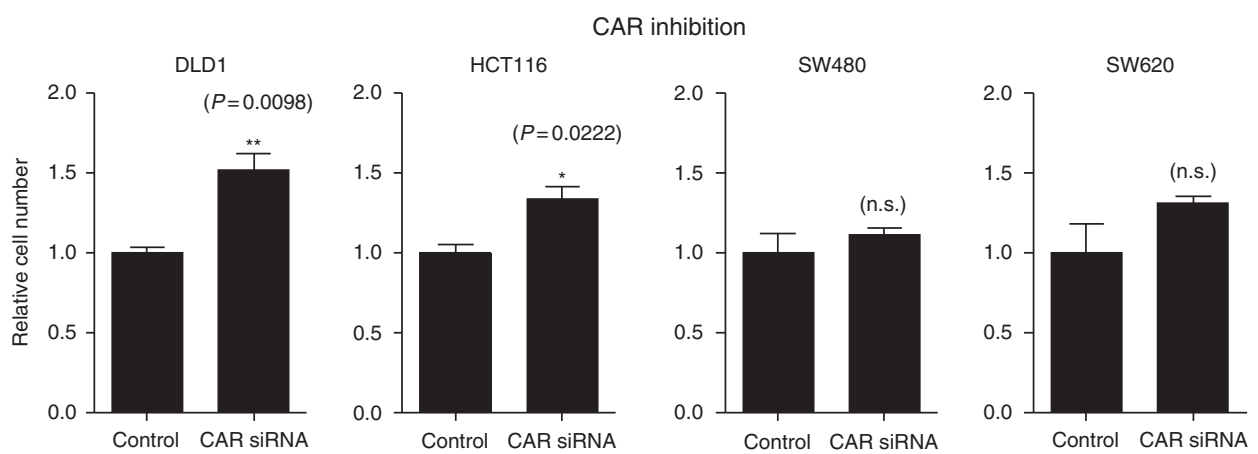

known to block CAR, markedly increased the invasiveness into matrigel of DlD1 and HCT116 cell lines compared with respective controls. However, SW480 and SW620 did not invade into matrigel neither with nor without RmcB (Figure 5A).

\section{Loss of CAR facilitates formation of liver metastases}

The impact of CAR inhibition on the metastatic behaviour of colon cancer cells was assessed in 6-week-old female athymic SCID-mice. At 4 weeks after intrasplenic injection, all those animals displayed liver metastases, which had received SW480 following siRNAmediated CAR knockdown. In contrast, liver metastases were found in only one animal (14\%) injected with SW480 control cells. Moreover, the number of liver metastases in the group with SW480 siRNA cells was significantly higher $(P=0.0169)$ (Figure $5 \mathrm{~B})$. In contrast, animals that had received injections of SW620 with upregulated CAR or controls did not display liver metastases (data not shown).

\section{CAR facilitates anti-apoptotic effects in colon cancer cells}

The potential effect of CAR on the response of colon cancer cells to apoptotic stimuli was tested by treatment with TRAIL. Hereby, significantly higher caspase 3/7 levels were found in SW480, DlD1, and HCT116. In the SW620 cell line, a minor increase was noted only. Enforced CAR expression led to lower caspase 3/7 activity in DLD1 cells upon treatment with TRAIL, whereas no changes were found in HCT116 and SW620, and an increase in the SW480 cell line was found (data not shown).

\section{DISCUSSION}

The current study marks the first report of differential expression and subcellular distribution of CAR in the adenoma-carcinoma sequence of colon cancer. Our observations reveal an increase of CAR mRNA expression in adenomas and a downregulation in primary cancers and metastasis. On a subcellular level we noted (1) a significant increase of cytoplasmic CAR in neoplastic tissues compared with the normal mucosa, (2) reduced CAR presence at the plasma membrane in primary cancers, and (3) a 're-localisation' of CAR to the plasma membrane in metastatic tumours. In conjunction with our data regarding the effects of CAR on growth, invasion, metastasis, and response to apoptotic stimuli, it may be speculated that CAR facilitates complex, stage-dependent functions in the course of colon carcinogenesis.

Our finding of high CAR mRNA expression and CAR protein localisation at the plasma membrane and within the cytoplasm in adenomas is in line with previous observations in early-stage breast cancer, Barrett's oesophagus, and early-stage oesophageal adenocarcinoma (Anders et al, 2003b; Anders et al, 2003b, 2009a). Therefore, it may be speculated that high CAR expression marks are a more general phenomenon in pre- and early malignant lesions. Functionally, CAR may promote early carcinogenesis as suggested for cervix and ovarian cancers, with CAR-expressing cell lines displaying less sensitivity towards apoptotic stimuli (Bruning et $a l, 2005)$. In line with this hypothesis, we found that colon cancer cell lines become more sensitive towards treatment with TRAIL following RNAi-mediated functional CAR knockdown. However, following CAR upregulation, either no change in caspase activity or contrary results were observed. Therefore, it remains unclear whether CAR contributes to the suppression of apoptosis in colonic neoplasias (Tsujitani et al, 1996).

Our observation of reduced CAR mRNA expression and CAR protein presence at the plasma membrane in primary colon cancers compared with non-transformed tissue is in agreement with reports for primary colon cancers and several other carcinomas (Heideman et al, 2001; Rauen et al, 2002; Sachs et al, 2002; Matsumoto et al, 2005; Korn et al, 2006; Buscarini et al, 2007; 
A
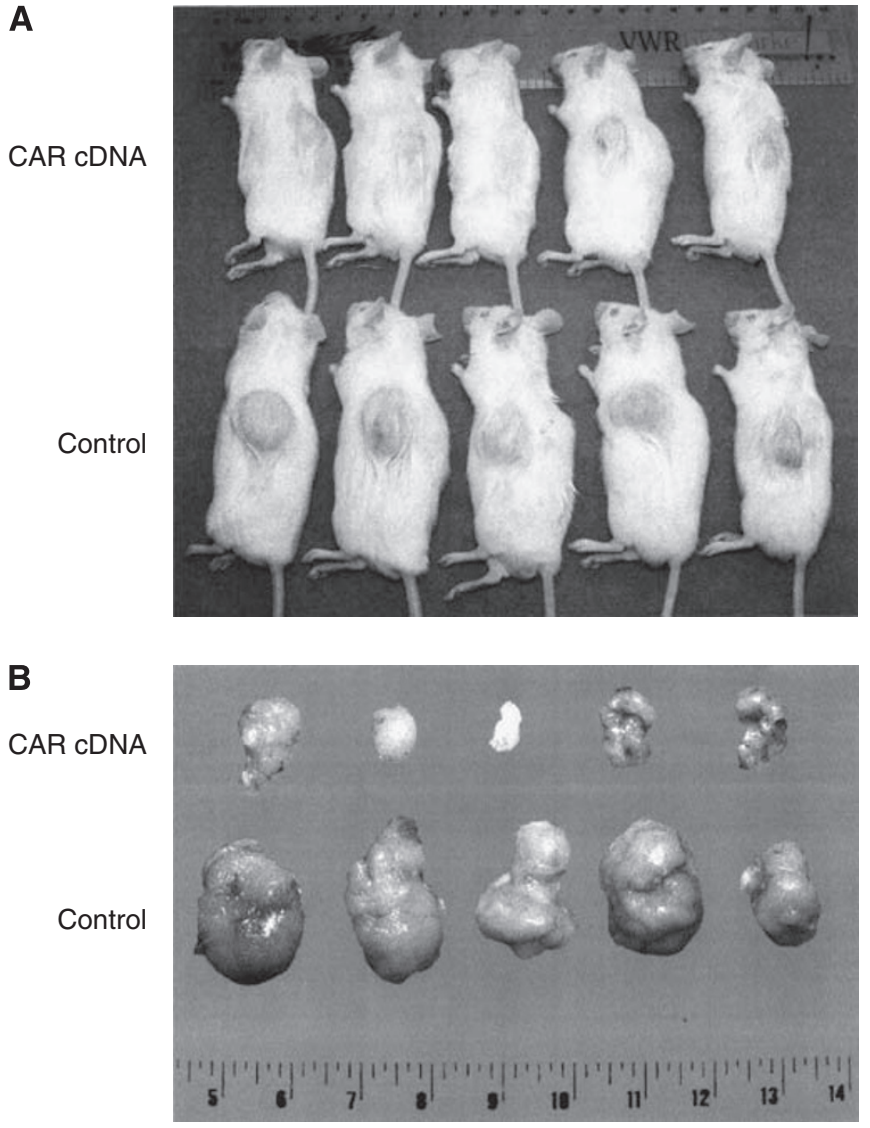

C

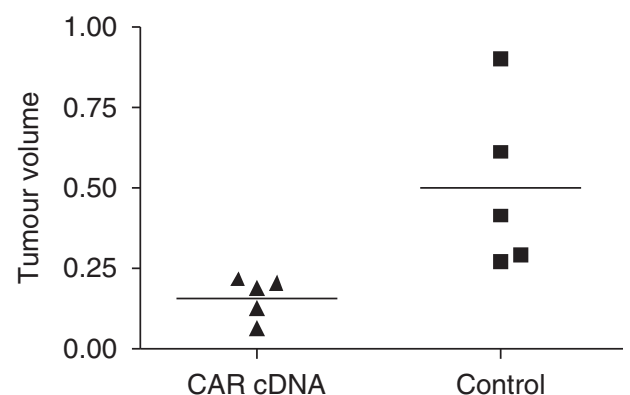

Figure 4 Impact of CAR on colon cancer growth in vivo. Ectopic CAR expression in SW480 cells resulted in a marked size reduction of xenograft tumours shown in situ $(\mathbf{A})$ and freshly explanted $(\mathbf{B})$. Determination of tumour volumes revealed a statistically significant difference between CAR overexpressing SW480 cells compared with the 'vector only' control cell line $(\mathbf{C})$.

Zhang et al, 2008; Anders et al, 2009b). These findings are highly suggestive of an entity-independent downregulation of CAR in primary carcinomas. The loss of CAR at the plasma membrane may led to an impaired intercellular adhesion as prerequisite for cancer dissemination as suggested for ovarian, bladder, and gastric cancers as well as gliomas (Okegawa et al, 2001; Bruning and Runnebaum, 2004; Huang et al, 2005; Wang et al, 2005; Anders et al, 2009b). In accordance with this hypothesis, we found that blocking of the extracellular portion of CAR increases the invasiveness of colon cancer cells. Furthermore, we observed a marked rise in the metastatic behaviour of colon cancer cells following CAR knockdown. These findings are in line with a previous report showing reduced potential of murine CT26 cells to form lung metastases (Yamashita et al, 2007). In conjunction with our observations in human tissues, these data suggest that reduced CAR expression in primary colon cancers contributes to the spreading of colon cancer cells and therefore to disease progression.

Furthermore, our findings show that CAR is involved in the regulation of colon cancer cell proliferation. We noted reduced growth of colon cancer cells upon ectopic CAR regulation in vitro and in vivo. In line with these findings, specific CAR silencing led to complementary effects as shown in vitro. These results are in agreement with previous observations in gastric, oesophageal, bladder, and prostate cancer cell lines, as well as glioma cells (Okegawa et al, 2000, 2001; Huang et al, 2005; Anders et al, $2009 \mathrm{a}, \mathrm{b})$, suggesting an entity-independent growth-suppressive function of CAR in human cancers. Underlying mechanisms, however, are not fully understood: Previously an accumulation of p21 and hypophosphorylated retinoblastoma protein upon CAR upregulation has been detected in human bladder cancer cell lines (Okegawa et al, 2001). On the basis of these findings, it has been hypothesised that cell-cell contact, initiated by membrane-bound CAR, may elicit a negative signal cascade modulating cell cycle regulators.

In colon cancer metastases, we noted abundant CAR immunopositivity confirming findings of a previous study describing $\sim 60 \%$ of colon cancer metastases in the liver to express CAR (Korn et al, 2006). Our findings are also in agreement with observations in local and distant metastases of prostate cancer, which present a strong membranous staining comparable to the non-transformed prostate mucosa, which is also significantly higher than the one in primary prostate cancers (Rauen et al, 2002). Moreover, these data are in line with findings for the cytoplasmic TJ protein ZO-1, a known binding partner of CAR (Kaihara et al, 2003). Our findings also do suggest that CAR presence at the cell surface of metastatic cancers results from differential distribution within the cell and not from elevated gene expression. Whether regulatory pathways such as Raf/MEK/ERK signalling that has been demonstrated to impact CAR localisation in colon cancer cells before are involved, remains to be elucidated (Anders, 2003a). The function of CAR on colon cancer metastases, however, remains hypothetical. Previously, CAR presence has been shown to correlate with the state of differentiation in metastatic colon cancer (Korn et al, 2006). As it has been shown that colorectal cancers undergo dedifferentiation at the primary site and 're-differentiate' when metastastic to the liver (Brabletz et al, 2001; Kaihara et al, 2003). Reexpression of CAR may simply represent an epiphenomenon of cell differentiation. However, CAR may also be of functional importance as it might allow cancer cells to gain foothold at the metastatic site, as postulated for E-cadherin (De Marzo et al, 1999; Rubin et al, 2001; Liu et al, 2002). Recently, an isoform-specific localisation and regulation of CAR $\left(\mathrm{CAR}^{\mathrm{Ex} 7}\right.$ and $C A R^{\mathrm{Ex} 8}$ ) has been shown in human airway epithelia. Whether alternative splicing of the CAR gene occurs and impacts the development of colonic neoplasia, however, needs to be elucidated (Excoffon et al, 2010).

In conclusion, our results suggest that CAR mediates promoting as well as inhibiting functions in colon cancer biology, depending on the stage of the tumour development and progression. Interestingly, its inhibitory functions are in contrast to findings for another member of the TJ family, Claudin 1, which has been shown to promote cancer growth and metastatic properties (Dhawan et al, 2005). These findings underline the hypothesis of complex roles of $\mathrm{TJ}$ proteins in the process of colon cancer development.

\section{ACKNOWLEDGEMENTS}

We thank Dr J Bergelson (Division of Infectious Diseases, Children's Hospital of Philadelphia, Philadelphia, PA, USA) for providing the human full-length CAR cDNA expression vector 
A
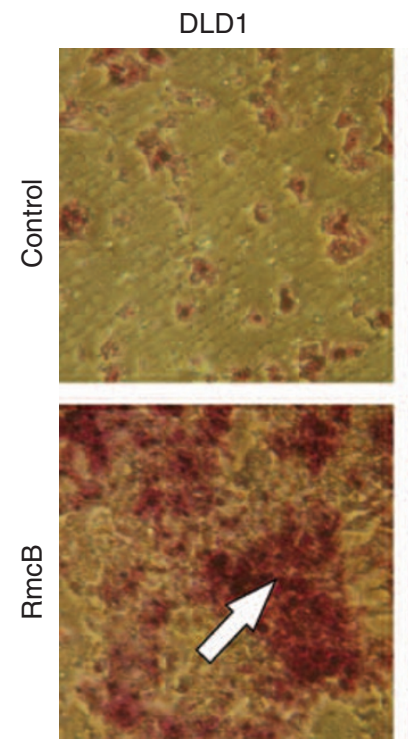

Invasion
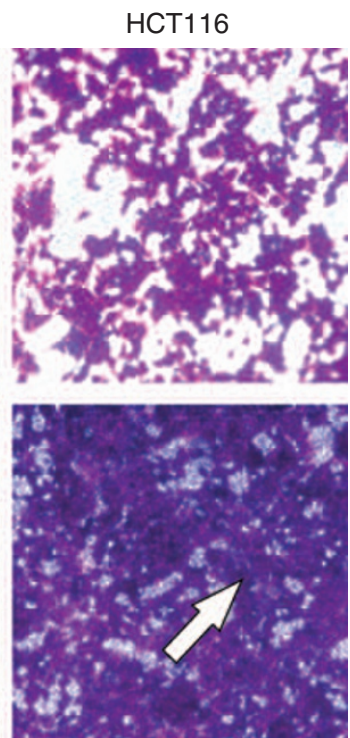

B Metastasis
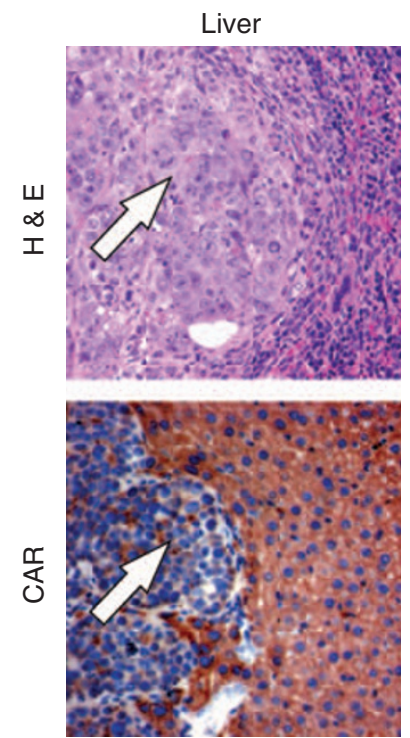

Figure 5 Inhibition of CAR promotes colon cancer cell invasiveness and metastasis. Blockade of the extracellular CAR portion using the anti-CAR antibody RmcB markedly increased the number of invading colon cancer cells in vitro (arrows) (A). RNAi-mediated knockdown of CAR in SW480 cells led to a significantly increased number of metastases (arrow) to the liver (lower panel demonstrates low CAR presence in metastatic cells (arrow) in contrast to adjacent CAR positive hepatic tissue) (B).

'hCARpcDNA3.1', the RmcB anti-CAR antibody, and CHO-CAR cells. Furthermore, we thank Dr C Nowak (Experimental Pharmacology \& Oncology GmbH, Berlin-Buch, Germany) and $\mathrm{K}$

\section{REFERENCES}

Anders M, Christian C, McMahon M, McCormick F, Korn WM (2003a) Inhibition of the Raf/MEK/ERK pathway up-regulates expression of the coxsackievirus and adenovirus receptor in cancer cells. Cancer Res 63(9): 2088-2095

Anders M, Hansen R, Ding RX, Rauen KA, Bissell MJ, Korn WM (2003b) Disruption of $3 \mathrm{D}$ tissue integrity facilitates adenovirus infection by deregulating the coxsackievirus and adenovirus receptor. Proc Natl Acad Sci USA 100(4): 1943-1948

Anders M, Rosch T, Kuster K, Becker I, Hofler H, Stein HJ, Meining A, Wiedenmann B, Sarbia M (2009a) Expression and function of the coxsackie and adenovirus receptor in Barrett's esophagus and associated neoplasia. Cancer Gene Ther 16(6): 508-515

Anders M, Vieth M, Rocken C, Ebert M, Pross M, Gretschel S, Schlag PM, Wiedenmann B, Kemmner W, Hocker M (2009b) Loss of the coxsackie and adenovirus receptor contributes to gastric cancer progression. $\mathrm{Br} J$ Cancer 100(2): 352-359

Bergelson JM, Cunningham JA, Droguett G, Kurt-Jones EA, Krithivas A, Hong JS, Horwitz MS, Crowell RL, Finberg RW (1997) Isolation of a common receptor for Coxsackie $B$ viruses and adenoviruses 2 and 5 . Science 275(5304): 1320-1323

Brabletz T, Jung A, Reu S, Porzner M, Hlubek F, Kunz-Schughart LA Knuechel R, Kirchner T (2001) Variable beta-catenin expression in colorectal cancers indicates tumor progression driven by the tumor environment. Proc Natl Acad Sci USA 98(18): 10356-10361

Bruning A, Runnebaum IB (2003) CAR is a cell-cell adhesion protein in human cancer cells and is expressionally modulated by dexamethasone, TNFalpha, and TGFbeta. Gene Therapy 10(3): 198-205

Bruning A, Runnebaum IB (2004) The coxsackie adenovirus receptor inhibits cancer cell migration. Exp Cell Res 298(2): 624-631

Bruning A, Stickeler E, Diederich D, Walz L, Rohleder H, Friese K, Runnebaum IB (2005) Coxsackie and adenovirus receptor promotes adenocarcinoma cell survival and is expressionally activated after transition from preneoplastic precursor lesions to invasive adenocarcinomas. Clin Cancer Res 11(12): 4316-4320
Atrott (Deutsches Herzzentrum, Berlin, Germany) for their support of the in vivo studies. This study was supported by a grant from the Deutsche Forschungsgemeinschaft to MA.
Buda A, Pignatelli M (2004) Cytoskeletal network in colon cancer: from genes to clinical application. Int J Biochem Cell Biol 36(5): $759-765$

Buscarini M, Quek ML, Gilliam-Hegarich S, Kasahara N, Bochner B (2007) Adenoviral receptor expression of normal bladder and transitional cell carcinoma of the bladder. Urol Int 78(2): 160-166

Cho KR, Vogelstein B (1992) Genetic alterations in the adenoma carcinoma sequence. Cancer 70(6 Suppl): 1727-1731

Cohen CJ, Shieh JT, Pickles RJ, Okegawa T, Hsieh JT, Bergelson JM (2001) The coxsackievirus and adenovirus receptor is a transmembrane component of the tight junction. Proc Natl Acad Sci USA 98(26): 15191 - 15196

Coyne CB, Voelker T, Pichla SL, Bergelson JM (2004) The coxsackievirus and adenovirus receptor interacts with the multi-PDZ domain protein-1 (MUPP-1) within the tight junction. J Biol Chem 279(46): 48079-48084

De Marzo AM, Knudsen B, Chan-Tack K, Epstein JI (1999) E-cadherin expression as a marker of tumor aggressiveness in routinely processed radical prostatectomy specimens. Urology 53(4): 707-713

Dhawan P, Singh AB, Deane NG, No Y, Shiou SR, Schmidt C, Neff J, Washington MK, Beauchamp RD (2005) Claudin-1 regulates cellular transformation and metastatic behavior in colon cancer. J Clin Invest 115(7): $1765-1776$

Excoffon KJ, Gansemer ND, Mobily ME, Karp PH, Parekh KR, Zabner J (2010) Isoform-specific regulation and localization of the coxsackie and adenovirus receptor in human airway epithelia. PLoS One 5(3): e9909

Excoffon KJ, Hruska-Hageman A, Klotz M, Traver GL, Zabner J (2004) A role for the PDZ-binding domain of the coxsackie $B$ virus and adenovirus receptor (CAR) in cell adhesion and growth. J Cell Sci 117(Part 19): 4401-4409

Grone J, Weber B, Staub E, Heinze M, Klaman I, Pilarsky C, Hermann K, Castanos-Velez E, Ropcke S, Mann B, Rosenthal A, Buhr HJ (2007) Differential expression of genes encoding tight junction proteins in colorectal cancer: frequent dysregulation of claudin-1, -8 and -12 . Int $J$ Colorectal Dis 22(6): 651-659

Hamilton SR, Aaltonen LA (2000) WHO Classification Tumours of the Digestive System Pathology \& Genetics. IARC Press: Lyon 
Heideman DA, Snijders PJ, Craanen ME, Bloemena E, Meijer CJ, Meuwissen SG, Van Beusechem VW, Pinedo HM, Curiel DT, Haisma HJ, Gerritsen WR (2001) Selective gene delivery toward gastric and esophageal adenocarcinoma cells via EpCAM-targeted adenoviral vectors. Cancer Gene Ther 8(5): 342-351

Huang KC, Altinoz M, Wosik K, Larochelle N, Koty Z, Zhu L, Holland PC, Nalbantoglu J (2005) Impact of the coxsackie and adenovirus receptor (CAR) on glioma cell growth and invasion: requirement for the C-terminal domain. Int $J$ Cancer 113(5): $738-745$

Kaihara T, Kawamata H, Imura J, Fujii S, Kitajima K, Omotehara F, Maeda N, Nakamura T, Fujimori T (2003) Redifferentiation and ZO-1 reexpression in liver-metastasized colorectal cancer: possible association with epidermal growth factor receptor-induced tyrosine phosphorylation of ZO-1. Cancer Sci 94(2): 166-172

Kimura Y, Shiozaki H, Hirao M, Maeno Y, Doki Y, Inoue M, Monden T, Ando-Akatsuka Y, Furuse M, Tsukita S, Monden M (1997) Expression of occludin, tight-junction-associated protein, in human digestive tract. Am J Pathol 151(1): 45-54

Korn WM, Macal M, Christian C, Lacher MD, McMillan A, Rauen KA, Warren RS, Ferrell L (2006) Expression of the coxsackievirus- and adenovirus receptor in gastrointestinal cancer correlates with tumor differentiation. Cancer Gene Ther 13(8): $792-797$

Kuster K, Grotzinger C, Koschel A, Fischer A, Wiedenmann B, Anders M (2010) Sodium butyrate increases expression of the coxsackie and adenovirus receptor in colon cancer cells. Cancer Invest 28(3): 268-274

Liu J, Ikeguchi M, Nakamura S, Kaibara N (2002) Re-expression of the cadherin-catenin complex in lymph nodes with metastasis in advanced gastric cancer: the relationship with patient survival. J Exp Clin Cancer Res 21(1): 65-71

Matsumoto K, Shariat SF, Ayala GE, Rauen KA, Lerner SP (2005) Loss of coxsackie and adenovirus receptor expression is associated with features of aggressive bladder cancer. Urology 66(2): $441-446$

Mirza M, Raschperger E, Philipson L, Pettersson RF, Sollerbrant K (2005) The cell surface protein coxsackie- and adenovirus receptor (CAR) directly associates with the ligand-of-numb protein-X2 (LNX2). Exp Cell Res 309(1): $110-120$

Miwa N, Furuse M, Tsukita S, Niikawa N, Nakamura Y, Furukawa Y (2000) Involvement of claudin- 1 in the beta-catenin/Tcf signaling pathway and its frequent upregulation in human colorectal cancers. Oncol Res 12(11-12): $469-476$

Okegawa T, Li Y, Pong RC, Bergelson JM, Zhou J, Hsieh JT (2000) The dual impact of coxsackie and adenovirus receptor expression on human prostate cancer gene therapy. Cancer Res 60(18): 5031-5036

Okegawa T, Pong RC, Li Y, Bergelson JM, Sagalowsky AI, Hsieh JT (2001) The mechanism of the growth-inhibitory effect of coxsackie and adenovirus receptor (CAR) on human bladder cancer: a functional analysis of car protein structure. Cancer Res 61(17): 6592-6600

Okegawa T, Sayne JR, Nutahara K, Pong RC, Saboorian H, Kabbani W, Higashihara E, Hsieh JT (2007) A histone deacetylase inhibitor enhances adenoviral infection of renal cancer cells. J Urol 177(3): 1148-1156

Parkin DM, Bray F, Ferlay J, Pisani P (2005) Global cancer statistics, 2002. CA Cancer J Clin 55(2): 74-108

Qin M, Escuadro B, Dohadwala M, Sharma S, Batra RK (2004) A novel role for the coxsackie adenovirus receptor in mediating tumor formation by lung cancer cells. Cancer Res 64(18): 6377-6380
Raschperger E, Thyberg J, Pettersson S, Philipson L, Fuxe J, Pettersson RF (2006) The coxsackie- and adenovirus receptor (CAR) is an in vivo marker for epithelial tight junctions, with a potential role in regulating permeability and tissue homeostasis. Exp Cell Res 312(9): 1566-1580

Rauen KA, Sudilovsky D, Le JL, Chew KL, Hann B, Weinberg V, Schmitt LD, McCormick F (2002) Expression of the coxsackie adenovirus receptor in normal prostate and in primary and metastatic prostate carcinoma: potential relevance to gene therapy. Cancer Res 62(13): $3812-3818$

Resnick MB, Gavilanez M, Newton E, Konkin T, Bhattacharya B, Britt DE, Sabo E, Moss SF (2005) Claudin expression in gastric adenocarcinomas: a tissue microarray study with prognostic correlation. Hum Pathol 36(8): $886-892$

Rubin MA, Mucci NR, Figurski J, Fecko A, Pienta KJ, Day ML (2001) E-cadherin expression in prostate cancer: a broad survey using highdensity tissue microarray technology. Hum Pathol 32(7): 690-697

Sachs MD, Rauen KA, Ramamurthy M, Dodson JL, De Marzo AM, Putzi MJ, Schoenberg MP, Rodriguez R (2002) Integrin alpha(v) and coxsackie adenovirus receptor expression in clinical bladder cancer. Urology 60(3): $531-536$

Satoh H, Zhong Y, Isomura H, Saitoh M, Enomoto K, Sawada N, Mori M. (1996) Localization of 7H6 tight junction-associated antigen along the cell border of vascular endothelial cells correlates with paracellular barrier function against ions, large molecules, and cancer cells. Exp Cell Res 222(2): $269-274$

Soler AP, Miller RD, Laughlin KV, Carp NZ, Klurfeld DM, Mullin JM (1999) Increased tight junctional permeability is associated with the development of colon cancer. Carcinogenesis 20(8): 1425-1431

Sollerbrant K, Raschperger E, Mirza M, Engstrom U, Philipson L, Ljungdahl PO, Pettersson RF (2003) The Coxsackievirus and adenovirus receptor (CAR) forms a complex with the PDZ domain-containing protein ligandof-numb protein-X (LNX). J Biol Chem 278(9): 7439-7444

Stecker K, Koschel A, Wiedenmann B, Anders M (2009) Loss of coxsackie and adenovirus receptor downregulates alpha-catenin expression. $\mathrm{Br} J$ Cancer 101(9): 1574-1579

Tsujitani S, Shirai H, Tatebe S, Sugamura K, Ohfuji S, Gomyo Y, Maeta M, Ito H, Kaibara N (1996) Apoptotic cell death and its relationship to carcinogenesis in colorectal carcinoma. Cancer 77(8 Suppl): $1711-1716$

Tsukita S, Furuse M (2000) Pores in the wall: claudins constitute tight junction strands containing aqueous pores. J Cell Biol 149(1): 13-16

Tsukita S, Furuse M, Itoh M (1996) Molecular dissection of tight junctions. Cell Struct Funct 21(5): $381-385$

Wang B, Chen G, Li F, Zhou J, Lu Y, Ma D (2005) Inhibitory effect of coxsackie adenovirus receptor on invasion and metastasis phenotype of ovarian cancer cell line SKOV3. J Huazhong Univ Sci Technolog Med Sci 25(1): $85-87,93$

Wittekind C (2003) TNM Klassifikation maligner Tumoren. 6th ed

Yamashita M, Ino A, Kawabata K, Sakurai F, Mizuguchi H (2007) Expression of coxsackie and adenovirus receptor reduces the lung metastatic potential of murine tumor cells. Int J Cancer 121(8): 1690-1696

Zhang NH, Song LB, Wu XJ, Li RP, Zeng MS, Zhu XF, Wan DS, Liu Q, Zeng YX, Zhang XS (2008) Proteasome inhibitor MG-132 modifies coxsackie and adenovirus receptor expression in colon cancer cell line lovo. Cell Cycle 7(7): $925-933$ 